Andreas Richter*, Maxim Scheck, Tobias Gehling, Christian Bohn, Volker Wesling und Christian Rembe

\title{
Erfassung geometrischer Daten des Schmelzbades zur Regelung eines WAAM-Prozesses
}

\author{
Acquisition of geometric data of the weld pool for controlling a WAAM process
}

DOI 10.1515/teme-2021-0072

Zusammenfassung: Als Möglichkeit individuelle oder komplexe Produkte aus unterschiedlichen Materialien automatisiert, schnell und kostengünstig zu fertigen, gewinnt der 3D-Druck immer mehr an Bedeutung. Unter 3D-Druck werden mehre Verfahren zusammengefasst. Ein weit verbreitetes Verfahren ist das additive Aufbringen von Kunststoffschichten, um beispielsweise komplexe Modelle zu „drucken“. Dabei wird das erzeugte Produkt nicht durch subtraktive Fertigungsverfahren, wie Drehen oder Fräsen, erzeugt. Eine Möglichkeit Metalle additiv zu fertigen ist das „Wire and Arc Additive Manufacturing (WAAM)“ zur additiven Erzeugung einer Struktur. Die Anwendung von Lichtbogenschweißen als additives Verfahren stellt besondere Anforderungen an die Mess- und Regelungstechnik. Hier wird ein Messsystem vorgestellt, mit dem die Breite des Schmelzbades innerhalb einer Frequenz von $40 \mathrm{~Hz}$ bei einer Latenz von 25 ms erfasst wird und an einen Regler weitergegeben wird.

Schlüsselwörter: WAAM, Pyrometer, Lichtbogenschweißen, Geometriemessung, In-line-Geometriemessung.

Abstract: 3D printing is becoming increasingly important
as a way to manufacture individual or complex products
from different materials automatically, quickly and cost-
effectively. Several processes are summarised under 3D
printing. One widely used process is the additive appli-

*Korrespondenzautor: Andreas Richter, Lehrstuhl für Messtechnik, IEI - TU Clausthal, Leibnizstraße 28, 38678 Clausthal-Zellerfeld, E-Mail: richter@iei.tu-clausthal.de Christian Rembe, Lehrstuhl für Messtechnik, IEI - TU Clausthal, Leibnizstraße 28, 38678 Clausthal-Zellerfeld, E-Mail: rembe@iei.tu-clausthal.de

Maxim Scheck, Christian Bohn, Lehrstuhl für Regelungstechnik, IEI - TU Clausthal, Leibnizstraße 28, 38678 Clausthal-Zellerfeld, E-Mail: scheck@iei.tu-clausthal.de, bohn@iei.tu-clausthal.de Tobias Gehling, Volker Wesling, Lehrstuhl für Schweißtechnik, ISAF - TU Clausthal, Agricolastraße 2, 38678 Clausthal-Zellerfeld, E-Mail: office@isaf.tu-clausthal.de cation of plastic layers, for example to "print" complex models. In this case, the product created is not produced by subtractive manufacturing processes such as turning or milling. One possibility of manufacturing metals additively is "Wire and Arc Additive Manufacturing (WAAM)" for the additive creation of a structure. The use of arc welding as an additive process places special demands on measurement and control technology. In this paper, a measuring system is presented with which the width of the molten pool is recorded within a frequency of $40 \mathrm{~Hz}$ at a latency of $25 \mathrm{~ms}$ and passed on to a controller.

Keywords: WAAM, pyrometer, arc welding, geometry measurement, in-line geometry measurement.

\section{Einleitung}

Die additive Fertigung mittels Lichtbogenschweißen wird momentan intensiv erforscht $[3,9,13,16]$. Mit einem solchem Verfahren ist es, im Gegensatz zu subtraktiven Fertigungsverfahren möglich, komplexe Bauteile mit einem Minimum an Materialaufwand herzustellen. Klassisch wird Lichtbogenschweißen zum Fügen von Bauteilen genutzt. Bei dieser Verbindungstechnik kann von konstanten Parametern und Umgebungsbedingungen während des SchweiBens ausgegangen werden, wodurch der Prozess bereits durch eine einfache Steuerung stabil gehalten werden kann [17]. Bei einer additiven Fertigung reicht eine solche Steuerung nicht aus, da sich die Parameter des Prozesses und die Umgebungsbedingungen laufend ändern. Das „Wire and Arc Additive Manufacturing (WAAM)" ermöglicht den Druck komplexer Strukturen und folglich ändern sich die Umgebungsbedingungen wie z.B. die Wärmeabfuhr über die Struktur ständig. Die damit verbundene Erhöhung von Störeinflüssen führt zu besonderen Anforderungen an die Regelungstechnik. Die für die Regelungstechnik zu erfassenden Prozessparameter müssen in Echtzeit erfasst werden, was eine anspruchsvolle Aufgabe für die Messtech- 
nik darstellt. Die hier entworfene Messtechnik misst die Breite des Schmelzbades. Aus dem Schmelzbad resultiert die Schweißraupe und im mehrlagigen Zusammenhang die Geometrie des fertigen Produktes. Zudem bildet sich beim Abkühlen des Schmelzbades das Gefüge aus. Dieses Gefüge hat unmittelbaren Einfluss auf die Materialeigenschaften $[4,15]$. Aus diesen Gründen wird das Schmelzbad für eine Regelung vermessen.

Das Aufschmelzen in geringem Umfang des der zuvor gefertigten Struktur ist für die Gefügebildung über verschiedene Schweißraupen erforderlich [14], dies soll jedoch auf ein Minimum reduziert werden. Insbesondere bei der Fertigung kleiner Bauteile ist die Begrenzung der eingebrachten Energie wichtig, um den Wärmeeintrag zu minimieren. Beim WAAM ist das Schmelzbad kleiner als der Lichtbogen und darum muss der Sensor durch das Plasma des Lichtbogens messen können, ohne dass dadurch die Messwerte verfälscht werden. Die messtechnische Überwachung des Prozesses in unmittelbarer Nähe des Lichtbogens ist eine Herausforderung, da durch die Strahlung des Plasmas das Licht des glühenden Schmelzbads überstrahlt wird [11]. Der Sensor muss außerdem kompakt genug sein, damit er mit dem bewegten Schweißbrenner mitgeführt werden kann.

Chandrasekaran et.al. haben gezeigt, dass sich die geometrischen Größen bei klassischen Anwendungen von Lichtbogenschweißen durch die Prozessdaten wie Drahtvorschubgeschwindigkeit, Verfahrgeschwindigkeit oder dem Schutzgas mit hoher Wahrscheinlichkeit vorhersagen lassen [2]. Solche Modelle versagen allerdings bei der WAAMFertigung von komplexen Strukturbauteilen.

Bestard et. al. haben zur Erfassung der Schweißraupenbreite einen Laserscanner genutzt [1]. Die Abtastzeit betrug hier $20 \mathrm{~ms}$. Dieses System erfasst die Breite der Schweißraupe allerdings ein paar Zentimeter hinter dem Schmelzbad. Dieser Abstand führt zu einer zusätzlichen erheblichen Totzeit, welche das System ungeeignet für eine Regelungsaufgabe macht.

Font et. al. haben eine andere Möglichkeit entwickelt, die Breite eines Schmelzbades beim Plasmaschweißen zu messen [5]. Dabei wird das Schmelzbad mit einer Kamera aufgenommen und die Breite mittels Bildanalyse gemessen. Das Plasmaschweißen ähnelt dem Lichtbogenschweißen, allerdings wird hier gegenüber dem Lichtbogenschweißen durch eine gekühlte Gasdüse der Lichtbogen, sowie das Plasma, eingeschnürt. Die durch den Lichtbogen verursachten Störungen werden erheblich reduziert. Das System von Font et. al. muss keinen ausgebreiteten Lichtbogen ausblenden und ist dafür auch nicht ausgelegt. Aus diesem Grund ist dieses System nicht für eine Regelung der Schmelzbadbreite beim WAAM-Prozess geeignet.
Zhang et. al. hat zur Messung des Profils des Schmelzbades 2015 ein weiteres Verfahren vorgestellt. Dabei wird das Schmelzbad mit einem starken Laser mit einer Matrix von Laserpunkten angestrahlt. Die Reflexionen dieser Punkte werden auf einen Schirm dargestellt und von einer Kamera erfasst. Durch die Veränderung des Punktmusters kann die Form des Schmelzbades berechnet werden [18]. Das System ist für den hier dargestellten Prozess nicht geeignet, da wir eine Messtechnik für eine additive Fertigung benötigen. Das Messsystem von Zhang benötigt einen Raum geeigneter Größe, in dem Objekte die Strahlengänge nicht stören. Bei einer additiven Fertigung kann dies nicht gewährleistet werden. Zudem wird bei WAAM mit kleineren Schweißleistungen gearbeitet und es muss deshalb in der nähe des Drahts gemessen werden. Ein anderer Nachteil des Systems ist, dass die Grundplatte bewegt werden muss. Bei unserem Aufbau zum WAAM bewegt sich der Schweißbrenner und somit müsste der komplette Messaufbau mitbewegt werden.

Aus diesem Grund wird ein kompakter Sensor zur Messung der Breite des Schmelzbades vorgestellt. Scheck et al. [7] ermittelten Zeitkonstante des Schweißsystems mit $T_{\text {system }}=1 \mathrm{~s}$ daraus resultiert eine minimale Abtastrate von $2 \mathrm{~Hz}$. Um eine Filterung der Rohdaten zu ermöglichen wird eine Abtastrate von mindestens $40 \mathrm{~Hz}$ angestrebt, daraus resultiert eine Latenz von $25 \mathrm{~ms}$.

\section{Methoden}

\subsection{Messaufbau}

Das Messsystem basiert auf dem 2019 erstmalig vorgestellten, speziell angepassten Quotientenpyrometer [12]. Das Pyrometer unterdrückt das Spektrum des Plasmas, welches durch den Lichtbogen erzeugt wird, durch zwei $10 \mathrm{~nm}$ breite optische Bandpässe.

Dieses Pyrometer wird vom Schweißbrenner mitgeführt, somit ist des Schweißbad immer im selben Bereich der Aufnahme. Die Aufnahmen des Pyrometers werden von einem Algorithmus ausgewertet und die Temperaturverteilung im Schmelzbad bestimmt. In dieser Publikation wird gezeigt, dass Geometriedaten in Echtzeit aus den Aufnahmen extrahiert werden können, die an den Regler weitergeleitet werden. Der Regler berechnet aus der Differenz von Soll- und Istwert ein Stellsignal für die Schweißstromquelle. Die Schweißstromquelle passt die Prozesseinstellgrößen an die geforderte Schweißleistung an. Energie- und Materialeintrag pro Zeiteinheit werden entsprechend der in der Schweißstromquelle hinterlegten 
Kennlinien eingestellt. Daraus resultiert eine Änderung der Größe des Schmelzbades. Dieser Informationsfluss ist in Abbildung $1 \mathrm{zu}$ sehen.

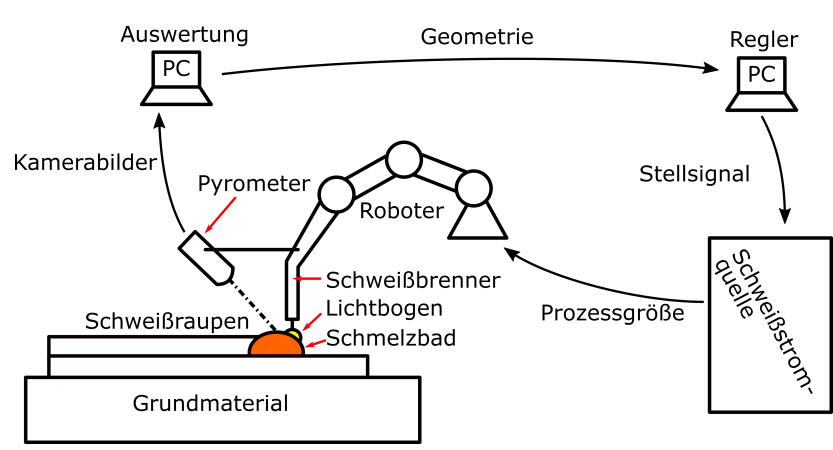

Abb. 1: Übersicht über das Gesamtsystem mit Informationsfluss.

Das Quotientenpyrometer misst die Temperatur des Schmelzbades über das Verhältnis der Strahlungsdichte an zwei verschieden Wellenlängen. Die Annahme eines grauen Strahlers als Strahlungsverhalten des Schmelzbades bildet die Grundlage dafür. Die Kameras in dem Pyrometer geben einen Helligkeitswert für jeden Pixel aus. Die Temperatur wird mit

$$
T_{\mathrm{G}_{\lambda_{1}}, \mathrm{G}_{\lambda_{2}}}=\frac{\left(\frac{1}{\lambda_{2}}-\frac{1}{\lambda_{1}}\right) C}{\ln \left(\frac{\mathrm{G}_{\lambda_{1}} \eta_{\lambda_{2}} \lambda_{2}}{\mathrm{G}_{\lambda_{2}} \eta_{\lambda_{1}} \lambda_{1}}\right)+5 \ln \left(\frac{\lambda_{1}}{\lambda_{2}}\right)}
$$

berechnet [12]. Die Gleichung 1 beinhaltet die Helligkeitswerte $\left(\mathrm{G}_{\lambda_{1}}, \mathrm{G}_{\lambda_{2}}\right)$, die Quanteneffizienzen der Kameras $\left(\eta_{\lambda_{1}}, \eta_{\lambda_{2}}\right)$, die Wellenlängen $\left(\lambda_{1}, \lambda_{2}\right)$ und eine Konstante $C=h c / k$, die mit dem Planck'schen Wirkungsquantum $h$, der Lichtgeschwindigkeit $c$ und der Boltzmann-Konstante $k$ berechnet wird. Die Wellenlängen werden durch zwei schmalbandige optische Filter vor den Kameras definiert. Die Filter sind so ausgewählt, dass sie das Plasma des Lichtbogens unterdrücken. In diesem Fall sind die Wellenlängen $\lambda_{1}=860 \mathrm{~nm}$ und $\lambda_{2}=680 \mathrm{~nm}$ ausgewählt worden.

\subsection{Auswertung der Daten}

Die Auswertung der Daten des Quotientenpyrometers ergibt eine Temperaturverteilung des Schmelzbades. Um die Latenz möglichst gering zu halten, wird die Datenverarbeitung innerhalb des Quotientenpyrometers effizient umgesetzt. Das Quotientenpyrometers arbeiten mit einer Lookup-Tabelle (LUT), in dieser Tabelle sind die
Temperaturen für die möglichen Kombinationen der Helligkeitswerte $G_{\lambda_{1}}$ und $G_{\lambda_{2}}$ aus Gleichung 1 hinterlegt [12]. Bei der hier betrachteten Messaufgabe ist eine genaue Kenntnis der Temperaturverteilung nicht notwendig. Aus diesem Grund wird die LUT entweder mit einer logischen 0 oder einer logischen 1 statt mit Temperaturewerten gefüllt. Dabei wird jeder Zelle der LUT mit einer Temperatur oberhalb der Liquidustemperatur eine logische 1 zugeordnet. Alle anderen Zellen erhalten eine logische 0 .

Das Ergebnis einer solchen Auswertung ist ein binäres Bild, dass zwischen flüssiger Schmelze und der bereits erstarrten Schmelze unterscheidet. Für die Auswertung wird die Region auf dem Bild ausgewählt, in der sich das Schmelzbad befindet. Dadurch werden Störungen die sich nicht in dieser Region befinden ignoriert. Nur die Daten aus dieser Region werden mit der LUT ausgewertet.

In dieser Region der Aufnahme werden nun Gruppen von Pixeln mit einer logischen 1 zusammengefasst. Um die Pixel zu gruppieren wird die IMAQ Particle Analysis VI von National Instruments genutzt [8]. Dieses Verfahren stammt ursprünglich aus der automatisierten Partikelanalyse. Dabei werden Pixel mit allen logischen 1 verbunden, die im direkten Umkreis liegen. Abbildung 2 stellt dies für ein einfaches Beispiel dar.

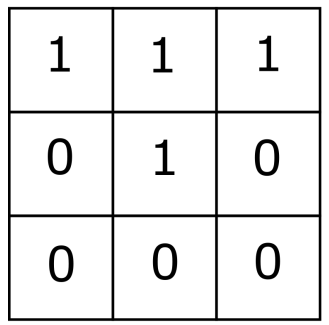

a)

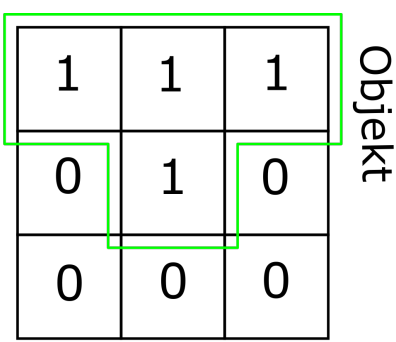

b)
Abb. 2: Zusammenfassung mehrere Pixel zu einem Objekt. a) Vor der Zusammenfassung zu einem Objekt. b) nach der Zusammenfassung zu einem Objekt. Das Objekt ist mit einer grüneren Linie umrandet.

Das größte zusammenhängende Objekt wird als Schmelzbad interpretiert. Somit wird vermieden, dass beispielsweise Funkenflug die Messung stört. Wenn Funkenflug oder Reflektionen an anderen Schweißraupen mitgemessen wird resultiert dies in einer Ausgabe von überhöhten Werten. Die Breite dieses Objektes wird als Breite des Schmelzbades in Pixeln interpretiert. In Abbildung 3 ist der Informationsfluss von den Helligkeitswerten der Kameras bis zur Ausgabe der Breite des Schmelzbades dargestellt.

Die Breite liegt zu diesem Zeitpunkt in einer Anzahl von Pixel vor. Zur Rückführung auf eine SI-Einheit wird das 


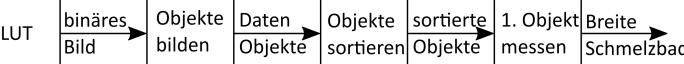

kann die Verzerrung der Abbildung durch den flacheren Blickwinkel ignoriert werden.

Abb. 3: Informationsfluss von den Helligkeitswerten des Kameras bis zur Breite des Schmelzbades.

Kamerabild mit einem USAF 1951 x1 target im Vorfeld vermessen. Damit erhält man einen Maßstab von $\beta=70 \mathrm{Pixel} / \mathrm{mm}$. Somit kann die gemessene Breite des Schmelzbades in Pixel $b_{\text {pix }}$ mit

$$
b_{\mathrm{mm}}=\frac{b_{\mathrm{pix}}}{\beta}
$$

auf die Breite des Schmelzbades in $\mathrm{mm} b_{\mathrm{mm}}$ umgerechnet werden.

\subsection{Versuchsaufbau}

Als Schweißzusatz wurde ISO 14341-A G 4Mo verwendet, das Material wurde auf Streifen aus S355JR mit den Abmessungen 300x40x15 mm aufgebracht. Die Abschirmung des Prozesses erfolgt über Schutzgas nach ISO 14175: M21ArC-18. Es wurden mehre Lagen übereinander geschweißt. Das Schmelzbad wurde mittels des Quotientenpyrometers während des Schweißvorgangs erfasst, die Auswertung der Rohdaten erfolgte auf einem Computer. In Abbildung 4 ist der Versuchsaufbau abgebildet. In der Versuchsreihe wurde mit relativ geringer Schweißleistung (ca. $4 \mathrm{~kW}$ ) geschweißt, um den Wärmeeintrag zu begrenzen. Um die Erfassung des entsprechend kleinen Schmelzbades trotz der Position unterhalb des Brenners zu gewährleisten, wurde das Pyrometer in einem flachen Winkel gegenüber dem Brenner montiert. Andernfalls verdeckt die Schutzgasdüse des Schweißbrenners das Schmelzbad je nach Schmelzbadgröße ganz oder teilweise.

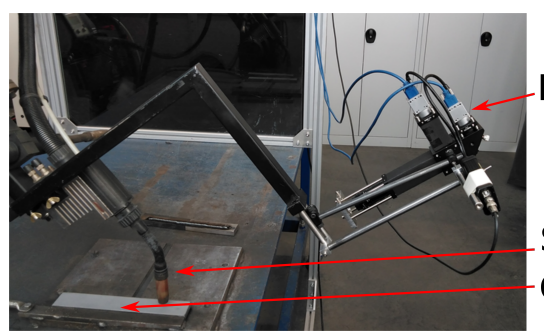

Pyrometer

Schweißbrenner Grundmaterial

Abb. 4: Versuchsaufbau.

In Abbildung 5 ist eine Aufnahme des Schmelzbades zu erkennen. Der Maßstab $\beta$ wurde für diese Versuche direkt am Schweißdraht ausgemessen. Aus diesem Grund

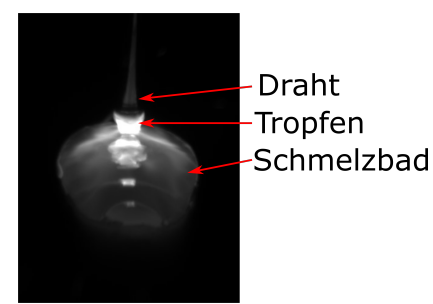

Abb. 5: Aufnahme des Schmelzbades aus dem Versuch bei 680 $\mathrm{nm}$.

Abbildung 5 zeigt das Schmelzbad der ersten Lage auf dem Metallstreifen.

\section{Ergebnisse}

Die Messung der Breite erfolgt nach dem vorher dargestellten Verfahren. Die Latenz der hier durchgeführten Messung beträgt $t_{l}=19 \mathrm{~ms}$. Die Messungen wurde mit einer Abtastrate von $f_{\text {mess }}=50 \mathrm{~Hz}$ durchgeführt. In Abbildung 6 ist eine Messung der Breite des Schmelzbades der ersten Lage aufgezeichnet.

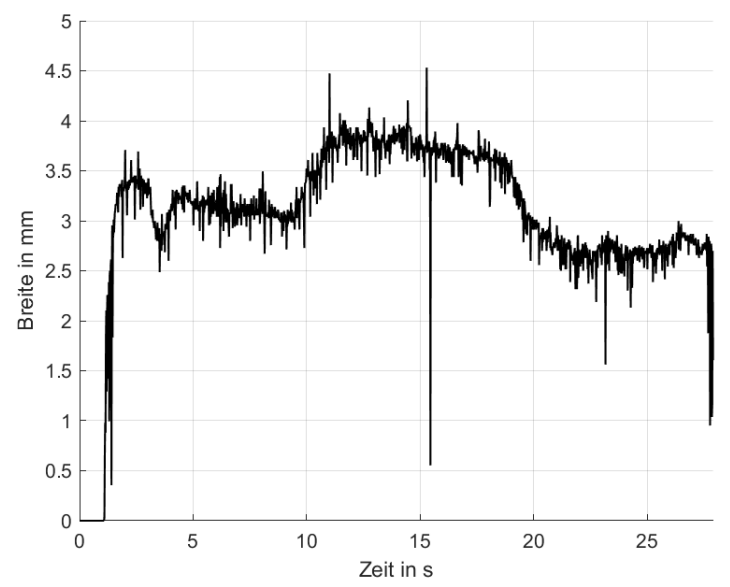

Abb. 6: Messung der Breite bei einem Schweißversuch.

Der Einbruch der Messung bei ungefähr 15,5 s ist durch eine Fehlzündung des Lichtbogens entstanden. Diese Fehlzündung ist in Abbildung 6 bei beiden genutzten Wellenlängen dargestellt.

Der genaue Grund, warum das Schmelzbad bei einer Wellenlänge von $680 \mathrm{~nm}$ zu einem großen Teil überstrahlt 

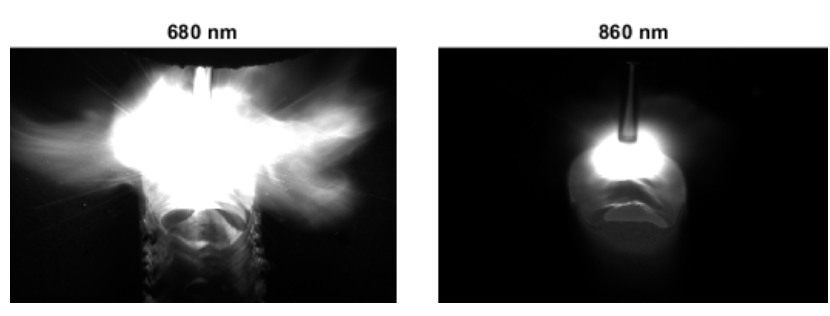

Abb. 7: Fehlzündung des Lichtbogens. Links bei $680 \mathrm{~nm}$ aufgenommen, rechts bei $860 \mathrm{~nm}$ aufgenommen.

wird, ist nicht bekannt. Das überblendete Gebiet kann nicht durch Hitze allein entstehen, da dies dann auch bei $860 \mathrm{~nm}$ zu erkennen sein müsste. Die optischen Filter sind so ausgewählt, dass die Wellenlängen des Plasmas, welche aus den beim Schweißen verwendeten Materialien entstehen, ausgeblendet werden. Die Überblendung kann durch das Eindringen eines fremden Materials in den Schweißprozess verursacht werden, beispielsweise durch Material, welches sich während der Schweißung an der Schutzgasdüse gesammelt hat.

Der hier verwendete Algorithmus für die Bestimmung der Schmelzbadbreite kann bei einer solchen Fehlaufnahme nur das letzte Stück des Schmelzbades als solches erkennen und daraus die Breite schätzen. Da das letzte Stück schmaler als der Rest des Schmelzbads ist, gibt der Algorithmus einen viel zu kleinen Schmelzbadbreite als Messergebnis zurück. Da solche Störungen nicht ausgeschlossen werden können, ist eine Filterung notwendig. Dazu wird ein Median über die letzten 3 Werte

$$
b_{\mathrm{med}}(k)=\operatorname{median}\left(b_{\mathrm{mm}}(k), b_{\mathrm{mm}}(k-1), b_{\mathrm{mm}}(k-2)\right)
$$

gebildet. Diese Filterung wird so schnell durchgeführt, dass eine Auswirkung auf die Latenz mit unseren Mitteln nicht messbar ist. Das Ergebnis der Daten aus Abbildung 6 mit dem Median ist in Abbildung 8 zu sehen.

\section{Zusammenfassung und Ausblick}

Das hier vorgestellte Verfahren eignet sich zur Erfassung der geometrischen Ausdehnung des Schmelzbades. So wurde eine einfache Möglichkeit geschaffen, die Breite des Schmelzbades im WAAM-Prozess in Echtzeit zu messen. Die Versuche wurden mit einer Abtastrate von $f_{\text {mess }}=50 \mathrm{~Hz}$ durchgeführt, die Latenz des Messsystems beträgt $t_{l}=19 \mathrm{~ms}$. Damit ist diese deutlich geringer als die Zeitkonstante $T_{\text {system }}=1 \mathrm{~s}$ des von Scheck et al. [7]

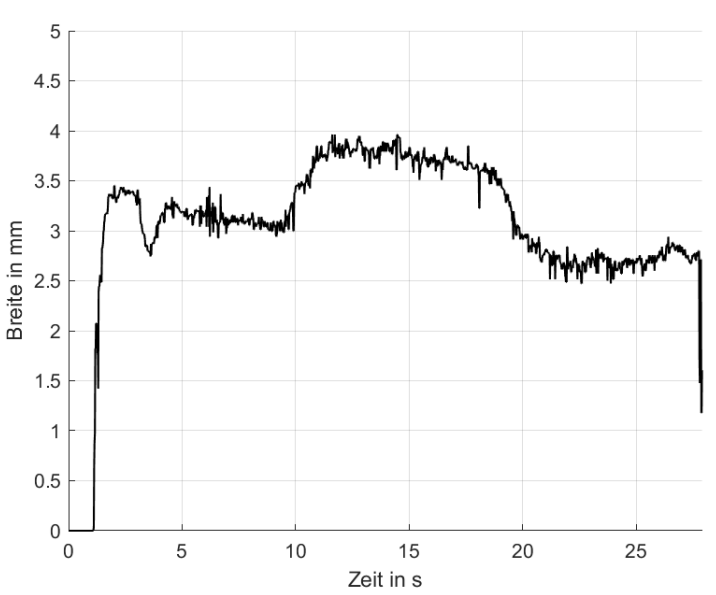

Abb. 8: Messung der Breite mit Median.

untersuchten Schweißprozesses. Der Sensor ermöglicht die online-Regelung eines WAAM-Prozesses.

Die Anwendung des Algorithmus' aus der Partikelanalyse, mit der die dem Schmelzbad zuordenbaren Pixel in der Aufnahme identifiziert werden, funktioniert stabil. Der Algorithmus interpretiert das größte flüssige Objekt als Schmelzbad. Damit wird eine fehlerhafte Messung beispielsweise durch Funkenflug oder Reflektionen verringert. Wenn durch äußere Einflüsse eine Auswertung der Bilder nicht möglich ist, liefert der Algorithmus keine sinnvollen Daten. Eine Filterung dieser Daten ist in der Lage, große Sprünge, die durch solche Störungen verursacht werden, zu unterdrücken. Durch eine weiter Reduzierung der Bandbreite der optischen Wellenlängenfilters, es es möglich, einen noch größeren Wellenlängenbereich zu unterdrücken. Damit werden die Einflüsse des Schweißlichtbogens noch weiter reduziert. Dies könnte dazu führen, dass der Einfluss von Fehlzündungen, wie in Abbildung 7, auf die Messung geringer wird.

Mit erweiterten Algorithmus könnte es möglich sein in Zukunft auch auf das komplette Volumen des Schmelzbades inklusive aufgeschmolzenen Grundmaterial zu schließen. Dazu könnte ein Finite Elemente-Modell nach Goldak et. al $[6,10]$ verwendet werden. Alternativ gibt es Ansätze dies bei klassischen Lichtbogenschweißen mit Fuzzylogic zu lösen [2]. Dies könnte auf WAAM versuchsweise angewendet werden.

\section{Literatur}

[1] G. A. Bestard, R. C. Sampaio, J. A. R. Vargas und S. C. A. Alfaro. Sensor Fusion to Estimate the Depth and Width of 
the Weld Bead in Real Time in GMAW Processes. Sensors (Basel, Switzerland), 18(4), 2018. 10.3390/s18040962.

[2] R. R. Chandrasekaran, M. J. Benoit, J. M. Barrett und A. P. Gerlich. Multi-variable statistical models for predicting bead geometry in gas metal arc welding. The International Journal of Advanced Manufacturing Technology, 105(1-4):15731584, 2019. ISSN 0268-3768. 10.1007/s00170-019-04355-0.

[3] X. Chen, C. Su, Y. Wang, A. N. Siddiquee, K. Sergey, S. Jayalakshmi und R. A. Singh. Cold Metal Transfer (CMT) Based Wire and Arc Additive Manufacture (WAAM) System. Journal of Surface Investigation: X-ray, Synchrotron and Neutron Techniques, 12(6):1278-1284, 2018. ISSN 1027-4510. 10.1134/S102745101901004X.

[4] U. Dilthey. Schweißtechnische Fertigungsverfahren 1: Schweiß- und Schneidtechnologien. VDI-Buch. SpringerVerlag Berlin Heidelberg, Berlin, Heidelberg, 3. bearbeitete Auflage, 2006. ISBN 9783540216735. 10.1007/3-540-33154-9. URL http://dx.doi.org/10.1007/3-540-33154-9.

[5] T. Font comas, C. Diao, J. Ding, S. Williams und Y. Zhao. A Passive Imaging System for Geometry Measurement for the Plasma Arc Welding Process. IEEE Transactions on Industrial Electronics, 64(9):7201-7209, 2017. ISSN 02780046. 10.1109/TIE.2017.2686349.

[6] J. Goldak, A. Chakravarti und M. Bibby. A new finite element model for welding heat sources. Metallurgical Transactions $B$, 15(2):299-305, 1984. ISSN 0360-2141. 10.1007/BF02667333.

[7] Maxim Scheck. Messung und Regelung im WAAM-Prozess. In Clausthal Centre for Material Technology, editor, 4. Symposium MATERIAL TECHNIK 25. bis 26. Februar 2021, Band 10, S. 140-150.

[8] NATIONAL INSTRUMENTS CORP. IMAQ Particle Analysis VI, 2021. URL https://zone.ni.com/reference/en-XX/help/ 370281AG-01/imaqvision/imaq_particle_analysis_report/.

[9] A. Nazir, K. M. Abate, A. Kumar und J.-Y. Jeng. A stateof-the-art review on types, design, optimization, and additive manufacturing of cellular structures. The International Journal of Advanced Manufacturing Technology, 104(9-12): 3489-3510, 2019. ISSN 0268-3768. 10.1007/s00170-01904085-3.

[10] M. R. Nezamdost, M. R. N. Esfahani, S. H. Hashemi und S. A. Mirbozorgi. Investigation of temperature and residual stresses field of submerged arc welding by finite element method and experiments. The International Journal of Advanced Manufacturing Technology, 87(1):615-624, 2016. ISSN 0268-3768. 10.1007/s00170-016-8509-4. URL https: //doi.org/10.1007/s00170-016-8509-4.

[11] Z. Pan, D. Ding, B. Wu, D. Cuiuri, H. Li und J. Norrish. Arc Welding Processes for Additive Manufacturing: A Review. In S. Chen, Y. Zhang und Z. Feng, editors, Transactions on Intelligent Welding Manufacturing, Band 81 of Transactions on Intelligent Welding Manufacturing, S. 3-24. Springer Singapore, Singapore, 2018. ISBN 978-981-10-5354-2. 10.1007/978-981-10-5355-9_1.

[12] A. Richter, C. Rembe, T. Gehling, K. Treutler und V. Wesling. Echtzeittemperaturmessung bei additivem Lichtbogenschweißen / Real-time temperature measurement at wire arc additive welding. tm - Technisches Messen, 86(s1):112-116, 2019. ISSN 0171-8096. 10.1515/teme-2019-0057.

[13] T. A. Rodrigues, V. Duarte, R. M. Miranda, T. G. Santos und J. P. Oliveira. Current Status and Perspectives on
Wire and Arc Additive Manufacturing (WAAM). Materials (Basel, Switzerland), 12(7), 2019. ISSN 1996-1944. $10.3390 / \mathrm{ma1} 2071121$.

[14] C. Schmid. Konstruktive Randbedingungen bei Anwendung des WAAM-Verfahrens. In R. Lachmayer, K. Rettschlag und S. Kaierle, editors, Konstruktion für die Additive Fertigung 2019, S. 203-222, Berlin, Heidelberg, 2020. Springer Berlin Heidelberg. ISBN 978-3-662-61149-4.

[15] V. Schuler und J. Twrdek. Praxiswissen Schweißtechnik: Werkstoffe, Prozesse, Fertigung. Springer Vieweg, Wiesbaden, 6 . vollständig überarbeitete Auflage, 2019. ISBN 9783658242657. 10.1007/978-3-658-24266-4. URL http://dx.doi.org/10.1007/978-3-658-24266-4.

[16] K. Treutler, S. Kamper, M. Leicher, T. Bick und V. Wesling. Multi-Material Design in Welding Arc Additive Manufacturing. Metals, 9(7):809, 2019. 10.3390/met9070809.

[17] B. Wang, S. J. Hu, L. Sun und T. Freiheit. Intelligent welding system technologies: State-of-the-art review and perspectives. Journal of Manufacturing Systems, 56:373391, 2020. ISSN 0278-6125. 10.1016/j.jmsy.2020.06.020. URL https://www.sciencedirect.com/science/article/pii/ S0278612520301102.

[18] W. J. Zhang, X. Zhang und Y. M. Zhang. Robust pattern recognition for measurement of three dimensional weld pool surface in GTAW. Journal of Intelligent Manufacturing, 26(4): 659-676, 2015. ISSN 0956-5515. 10.1007/s10845-013-0825$z$. 